\title{
Multiple Access Interference Mitigation Through Multi-Level Locally Orthogonal FH-CDMA
}

\author{
Jens Elsner, Ralph Tanbourgi and Friedrich K. Jondral \\ Karlsruhe Institute of Technology, Germany $\{$ jens.elsner, ralph.tanbourgi, friedrich.jondral $\} @$ kit.edu
}

\begin{abstract}
Multi-level locally orthogonal frequency hopping code division multiple access (MLLO-FH-CDMA) is introduced as a novel method to reduce self-interference in large scale $\mathbf{F H}$ CDMA ad hoc networks. It is analyzed in a stochastic geometry scenario and verified with simulations. The performance gains of multi-level hopping are shown by comparing it to standard FH-CDMA channel access.
\end{abstract}

\section{INTRODUCTION}

Frequency hopping code division multiple access (FHCDMA) systems are widely applied in military communications due to the inherent robustness against external interference created by, e.g., deliberate signal jamming and to decrease the probability of signal interception.

One aspect of critical importance in large scale ad hoc networks, as envisioned in military applications, is internal multiple access interference (MAI). The higher the number of users in the network, the more likely they are to interfere due to spatial reuse of the same frequency band. An important step to mitigate MAI is the introduction of multi-channel systems that operate in more than one hopping channel.

On the physical layer, these channels have to be organized in a way that the internal interference is minimized. Orthogonal multi-level hopping implements frequency planning by locally orthogonal frequency hopping, i.e. by assigning orthogonal hopping sequences to co-located nodes, to mitigate the internal interference dominated by nearby sources. It furthermore introduces multiple hopping layers for high number of users, resolving hot-spot situations where complete orthogonalization of neighboring transmissions is not possible as the number of communicating devices exceeds the number of available channels.

Adaptive FH in itself is, of course, not a novel concept. Other works treating various aspects of military and nonmilitary applications are, e.g., [1] or [2], [3]. [1] is one of the earlier analyzes of an adaptive frequency hopping scheme to combat jamming. Popovski et al. in [2], [3] evaluate two orthogonal hopping schemes that rely on frequency rolling and hop set splitting. Unfortunately, both schemes create deterministic collisions on the same channel if the number of nodes exceeds the number of channels and are hence not suitable for large scale networks that have to operate in the presence of external interference.

The main contribution of this paper is the introduction of the concept of multiple orthogonal hopping layers, where the collisions between layers are randomized, and its analysis. The resulting multi-level locally orthogonal FH-CDMA technique
(MLLO-FH-CDMA) can be applied as part of the PHY/MAC layer of a transmission protocol and significantly reduces MAI.

The remainder of this paper is structured as follows. Section II introduces the system model. Section III describes and analyzes orthogonal multi-level hopping and gives simulation results. Section IV concludes gives an outlook on future work.

\section{SYSTEM MODEL}

\section{A. Geometry, Channel and Receiver Model}

A network consists of nodes distributed in the plane. We assume that the nodes positions form a point process $\Pi \triangleq$ $\left\{X_{i}\right\}$. If we consider the network in one particular snapshot, there is a subset of transmitting nodes and a subset of receiving nodes. These nodes are labeled $X_{i}^{\mathrm{tx}} \in \Pi^{\mathrm{tx}} \subset \Pi$ and $X_{i}^{\mathrm{rx}} \in$ $\Pi^{\text {rx }} \subset \Pi$, respectively. Without loss of generality, we order the elements of $\left\{X_{i}^{\mathrm{tx}}\right\}$ and $\left\{X_{i}^{\mathrm{rx}}\right\}$ such that each transmitter $X_{i}^{\mathrm{tx}}$ has $X_{i}^{\mathrm{rx}}$ as its intended receiver. The distance separating every pair $X_{i}^{\mathrm{tx}}, X_{i}^{\mathrm{rx}}$ is assumed to be at most $r$ units.

If a node at position $X_{i}$ can cause strong ${ }^{1}$ interference to a node at position $X_{j}$, we say that these nodes are in a neighborhood and write $X_{i} \in \mathcal{N}_{j}$ and $X_{j} \in \mathcal{N}_{i}$, respectively. It is assumed that each node is aware of its neighborhood ${ }^{2}$.

The network bandwidth $B$ is the total bandwidth available for communication and is split into $M$ orthogonal channels of system bandwidth $B_{m}=\frac{B}{M}$, corresponding to the receiver bandwidth of a single node. Ambient noise power spectral density is $N_{0}$. The power attenuation factor between two points in the plane at distance $d>1$ is given by $d^{-\alpha}, \alpha>2$.

Each transmitter transmits with power $\rho$ and hence spectral power density $\frac{\rho}{B_{m}}=\frac{\rho M}{B}$ in a channel.

We consider transmissions in the resulting interference field and assume that a packet can be decoded if at the receiver a signal-to-noise-and-interference (SINR) threshold of $\beta$ is exceeded.

\section{B. Channel access}

We assume synchronized and slotted frequency hopping medium access, where, at a given instance, all nodes $X_{i}$ choose a certain channel $m_{i}, m_{i}=1, \ldots, M$.

The channels $m_{i}$ can be interpreted as marks of the points $X_{i}$, yielding a marked point process $\tilde{\Pi}$. The characteristics

\footnotetext{
${ }^{1}$ How exactly strong interference is defined depends on the features of the implemented communication protocol. E.g., a criterion can be that interference by $X_{i}$ is strong, if the receiver at $X_{j}$ is in outage with probability one.

${ }^{2}$ This can be realized by, e.g, periodical transmission of small beacon signals in a broadcast channel.
} 
of $\tilde{\Pi}$ strongly depend on the way the nodes choose their channel. E.g., for uncoordinated FH-CDMA, the point process $\tilde{\Pi}$ is independently marked, whereas for locally orthogonal hopping, $\tilde{\Pi}$ is dependently marked. In the latter case, the marks $m_{i}$ are spatially correlated, since the channel choice of a node $X_{i}$ depends on the channel choices of nodes within its neighborhood $\mathcal{N}_{i}$.

For locally orthogonal hopping as further analyzed in [4], the assignment of the marks corresponds to an allocation of the channels $m_{i}$ which is performed by a scheduling algorithm $\mathcal{S}$. We define $\tilde{\Pi}_{m} \triangleq\left\{\left(X_{i}, m_{i}\right) \mid X_{i} \in \Pi, m_{i}=m\right\}$ as the point process counting only those nodes which operate in channel $m$.

\section{ORthogonal Multi-Level Hopping}

\section{A. Principle}

Multiple access interference is dominated by nearby nodes transmitting in the same channel. In spatially locally orthogonal frequency hopping, neighboring nodes try to mitigate interference by choosing orthogonal hopping sequences. If there are $M$ channels, there exist $M$ orthogonal hopping sequences. Orthogonal hopping creates a spatial ordering that minimizes interference if there are enough channels available at every node in the network, i.e., if $\forall n:\left|\mathcal{N}_{n}\right| \leq M$ is true. If the number or nodes in a neighborhood exceeds the number of orthogonal hopping sequences, deterministic collisions of hopping sequences may follow. Unwanted collisions in the same channel can also occur if a certain node attracts more traffic than others, e.g., because they act as a gateway due to its spatial or hierarchical position. Introducing another hopping layer, i.e., another set of orthogonal hopping sequences that are weakly correlated with the first set, allows breaking the order where necessary to resolve these deterministic collisions. The interference of this new layer is then, from the perspective of any other layer, distributed fairly over all channels, while no interference occurs from nodes within the same layer.

Fig. 1 illustrates the principle for part of a network with a total of 11 nodes on three layers and four available channels. The figure shows the channel choice in the neighborhood of node 3, 4 and 5. As seen in the figure, the hopping sequences within a layer are locally orthogonal, i.e., no node occupies the same channel within the neighborhood indicated by colored circles. As there are more nodes than channels, more than one layer exists. A possible ${ }^{3}$ broadcast channel, indicated by $B$, is the same on all layers.

Note that if the number of hopping layers $L$ is equivalent to the number of nodes $N$, this scheme corresponds to standard FH-CDMA. If $L=1$, it corresponds to orthogonal hopping.

Of interest is now an algorithm $\mathcal{S}$ to reach a channel assignment according to the described MLLO-FH-CDMA scheme in a large scale ad hoc network.

\footnotetext{
${ }^{3}$ In multi-channel networks, several approaches for neighborhood discovery and transmission negotiation exist, some of which require a common broadcast channel. E.g., for asynchronous split phase protocols a shared channel is needed. Cf. [5], [6] and references therein.
}

\section{B. Channel assignment as a graph coloring problem}

In a large scale ad hoc network, decisions about channel assignment have to be made locally as every node has only a limited view on the whole network. Globally, the channel assignment problem can be interpreted as a graph coloring problem of an undirected graph $\mathcal{G}$. Two nodes are connected by an edge of $\mathcal{G}$ if they can be in conflict with each other, i.e. if they are in a neighborhood. The colors denote channels. For a given layer, a proper coloring of the conflict graph $\mathcal{G}$ corresponds to a valid channel assignment that allows for locally orthogonal hopping.

A fast converging distributed algorithm has to be given to reach a proper channel assignment according to the MLLO hopping scheme. The algorithm should converge to a solution that divides the (not necessarily colorable) graph $\mathcal{G}$ into a number of colorable subgraphs $\mathcal{G}_{1}, \ldots, \mathcal{G}_{L}$ corresponding to the hopping layers.

Two approaches for building the interference graph can be considered.

1) Receive channel scheduling for uncoordinated medium access: With receive channel scheduling, the receive channels are allocated in such a way that they are locally orthogonal. Transmitting nodes then choose the transmit channel according to the receive channel of the intended receiver. The corresponding conflicts are defined as follows: all nodes within the neighborhood $\mathcal{N}_{i}$ are in conflict with a node $X_{i}$. The vertex set of the interference graph $\mathcal{G}$ is hence $\left\{X_{i}\right\}$. An edge is placed between two nodes $X_{i}, X_{j}$, if $X_{j} \in \mathcal{N}_{i}$ or $X_{i} \in \mathcal{N}_{j}$.

2) Transmit and receive channel scheduling for CSMA/CAtype medium access: If the protocol allows for coordination of medium access, conflicts at both transmitter and receiver can be considered. In contrast to receive scheduling, this strategy is dynamic in the sense that it makes short-time allocations based on the knowledge of the actual transmission schedules. Here, a transmitter $X_{i}^{\text {tx }}$ is in conflict with all (unintended) receivers in its neighborhood $\mathcal{N}_{i}^{\text {tx }}$ and a receiver $X_{i}^{\text {rx }}$ is in conflict with all (unintended) transmitters in its neighborhood $\mathcal{N}_{i}^{\text {rx }}$. The additional labeling of the neighborhood is necessary to indicate the type of nodes (receiver or transmitters) creating a conflict. For instance, $\mathcal{N}_{i}^{\mathrm{rx}}$ is the set of transmitters in the neighborhood of the receiver $X_{i}^{\mathrm{rx}}$. The vertex set of the interference graph $\mathcal{G}$ is composed of all transmitter-receiver tuples $\left\{\left(X_{i}^{\mathrm{tx}}, X_{i}^{\mathrm{rx}}\right)\right\}$. An edge between two tuples $\left(X_{i}^{\mathrm{tx}}, X_{i}^{\mathrm{rx}}\right),\left(X_{j}^{\mathrm{tx}}, X_{j}^{\mathrm{rx}}\right)$ is drawn, if $X_{i}^{\mathrm{tx}} \in \mathcal{N}_{j}^{\mathrm{rx}}$ or $X_{i}^{\mathrm{rx}} \in \mathcal{N}_{j}^{\mathrm{tx}}$.

Fig. 1 illustrates the two approaches to create the interference graph.

Transmit and receive scheduling will naturally lead to better interference avoidance than receive channel scheduling, as the actual physical conflicts are considered. However, it requires a possibly network-wide negotiation phase before each transmit phase. Receive channel scheduling has the advantage that no re-negotiation of channels is needed, even if the communication partner changes and hence creates less protocol overhead. It can be a good choice for static or slowly changing network topologies. 

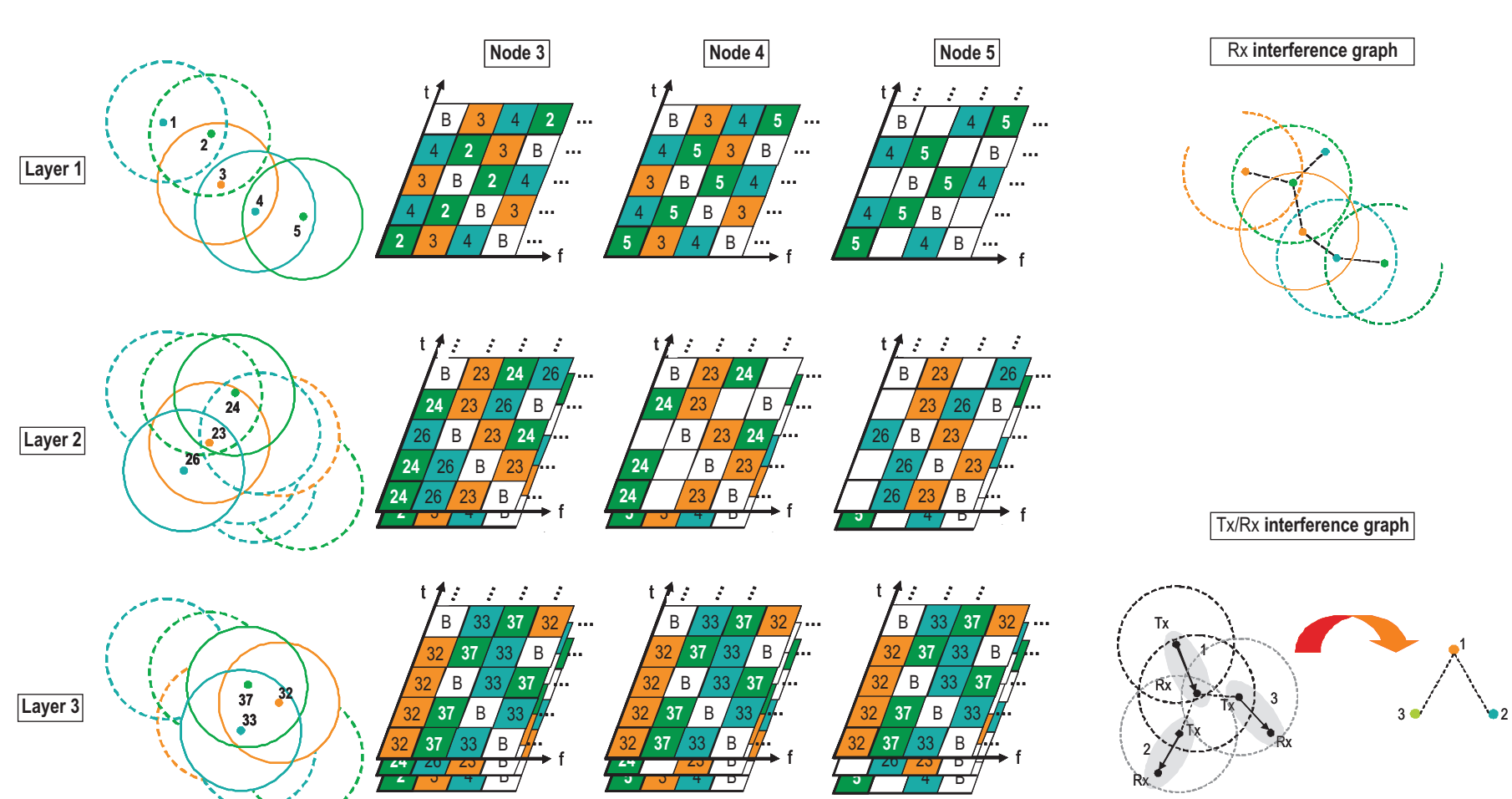

Fig. 1. Principle of multi-level orthogonal frequency hopping

In the following, we will focus on receive channel scheduling, but note that the principal approach is the same if CSMA/CA-type medium access is employed.

3) Distributed multi-level coloring for receive channel scheduling: With $M$ channels, a layer $\mathcal{G}_{i}$ is surely colorable if any node in that layer has no more than $M-1$ conflicts (Greedy coloring) ${ }^{4}$.

As an example of an algorithm leading to a proper MLLO channel assignment, we propose the following algorithm ${ }^{5}$, extending [8], [9]:

Each node selects a channel $m$ in a layer $\mathcal{G}_{i}$ according to a probability distribution $p_{n}(t)$, kept by each node. Each node furthermore keeps a hop sequence collision counter $k_{n}(t)$ and a collision-free counter $\bar{k}_{n}(t)$.

- Upon entering the network, a node starts with a search hop sequence uncorrelated to all layers. In this way, the quality of all channels and the neighborhood can be acquired.

- Once the neighborhood is known, the node starts in the first layer and initializes $p_{n}(t)$ to a discrete uniform distribution.

- The probability distribution is evolved according to the update rule (1). Each time a node experiences a conflict, $k_{n}(t)$ is increased and $\bar{k}_{n}(t)$ is reset to zero. If a node has no conflict, $\bar{k}_{n}(t)$ is increased and $k_{n}(t)$ is reset to zero.

\footnotetext{
${ }^{4}$ For most graphs, even $M$ neighbors result in a surely colorable graph, $\mathrm{cf}$. Brooks' Theorem [7].

${ }^{5}$ Of course, other distributed coloring algorithms can be used to implement MLLO hopping as well.
}

- If the collision counter of a node exceeds a threshold $\zeta$, $k_{n}(t)>\zeta$, the node moves up a layer and sets $p_{n}(t)$ to a discrete uniform distribution.

- If the collision-free counter of a node exceeds a threshold $\epsilon, \bar{k}_{n}(t)>\epsilon$, and the node is not in the first layer, the layer below is checked. If there are less than $M$ nodes in the neighborhood on the layer below, the node moves down a layer and set $p_{n}(t)$ to a discrete uniform distribution.

- If the packet error rate exceeds a threshold $\tau$, the node chooses a hop sequence uncorrelated to all layers.

The update rule for channel selection is

$$
p_{n}(t+1)= \begin{cases}\delta_{c_{n}(t)} & , \forall i \in \mathcal{N}_{n}: c_{n}(t) \neq c_{i}(t) \\ (1-\gamma) p_{n}(t) & \\ +\frac{\gamma}{M-1} \bar{\delta}_{c_{n}(t)} & , \text { otherwise }\end{cases}
$$

where $\delta_{c_{n}(t)}$ denotes the vector of length $M$ with a one at position $c_{n}(t)$ and a zero otherwise; $\bar{\delta}_{c_{n}(t)}$ denotes the vector of length $M$ with a zero at position $c_{n}(t)$ and a one otherwise.

The algorithm is parameterized by the resistance to change a channel if in conflict on the same layer $\gamma \in(0,1)$, the resistance to move up a layer $\zeta$, the resistance to move down a layer $\epsilon$ and a bail-out threshold $\tau$. At each node, $\delta$ and $\epsilon$ should be randomized to avoid deterministic collisions.

We note that this algorithm will lead to a MLLO channel assignment, but not necessarily to a global optimum. The resulting channel assignment might not be globally optimal, since interference is also influenced by the activity and position of nodes inside and outside the neighborhood. The interference 
graph can be thought of a being complete (fully connected, since every node influences every other node) and weighted by the interference potential. The method described here can - and should - be extended in a practical protocol by including the channel quality (e.g. by measuring the per channel packet error rate and determined by position and activity of other nodes) due to non-decodable internal or external interference at each node when choosing a channel and layer. The protocol should tend to group very active and close nodes in a neighborhood in the same layer to reduce MAI.

In the algorithm, nodes exposed to stronger interference due to their position or activity resort to uncorrelated hopping if a packet error rate threshold of $\tau$ is exceeded. Note that if $\tau=0$, the scheme again corresponds to standard FH-CDMA.

\section{Analysis}

In the following, we shall evaluate the improvement of MLLO hopping compared to uncoordinated FH-CDMA using a simplified scenario. The point process $\Pi$ introduced in section II is assumed to be a Binomial process with $N$ nodes on a bounded Region $W$, i.e., $\Pi^{(N)} \triangleq\left\{X_{0}, X_{1}, \ldots, X_{N-1}\right\}$, where all $X_{i} \in W \subset \mathbb{R}^{2}$. An interference graph is then constructed by drawing edges between all pairs $X_{i}, X_{j}$, with $i \neq j$. Such a construction models the interference situation in a neighborhood, where every node's transmission creates excessive interference to the other nodes, directly creating outage.

We further introduce the following quantities:

- The activity indicators, denoting the probability of transmission of a node, are given by $\left\{a_{0}, a_{1}, \ldots, a_{N-1}\right\}$, where the $a_{i}$ are i.i.d. in the interval $[0,1]$ and have mean $\bar{a}$.

- The collision indicators are given by $\left\{\kappa_{i j}\right\}$, with $i, j=$ $0, \ldots, N-1, i \neq j$. Formally, $\kappa_{i j}=1$ indicates the event that nodes $X_{i}$ and $X_{j}$ are in conflict, i.e., they have chosen the same channel. The complementary event is given by $\kappa_{i j}=0$, thus $\kappa_{i j} \in\{0,1\}$.

By considering the $a_{i}$ as marks associated with the points $X_{i}$, we define by $\tilde{\Pi}^{(N)} \triangleq\left\{\left(X_{1}, a_{1}\right), \ldots,\left(X_{N-1}, a_{N-1}\right)\right\}$ the marked Binomial point process. To investigate the interference situation within this network, it is necessary to consider the network from the viewpoint of a specific point. Therefore, we condition $\tilde{\Pi}^{(N)}$ on having the point $X_{0}$ in the origin and are interested in the interference situation at this node. When a point process with distribution $\mathbf{P}$ is conditioned on having a point in the origin without counting it, the reduced Palm distribution $\mathbf{P}^{! o}$ must be used to further analyze the point process. From Slivnyak's theorem [10], it follows that $\mathbf{P}^{! o}\left(\tilde{\Pi}^{(N)}(W)\right)=\mathbf{P}^{o}\left(\tilde{\Pi}^{(N)}(W) \backslash X_{0}\right)=\mathbf{P}\left(\tilde{\Pi}^{(N-1)}\right)$ for a Binomial process. This means, the statistics of $\tilde{\Pi}^{(N)}$ conditioned on having a point in the origin without counting it, are the same as the statistics of $\tilde{\Pi}^{(N-1)}$ which is essentially due to the independence among both the $X_{i}$ and $a_{i}$. The point

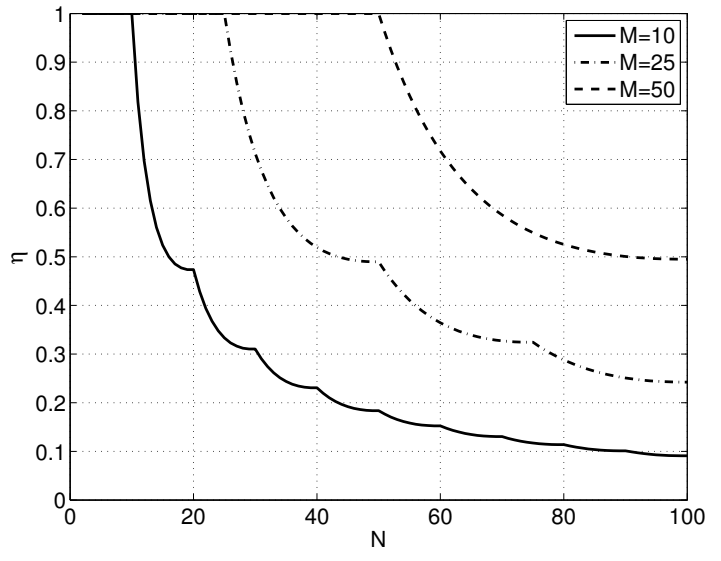

Fig. 2. Relative gain of MLLO-FH-CDMA in terms of relative reduction of average conflicts.

$X_{0}$ is referred to as the reference node ${ }^{6}$.

The comparison between MLLO hopping and FH-CDMA will be based on the mean number of conflicts the node $X_{0}$ experiences ${ }^{7}$.

1) Case FH-CDMA: The average number of conflicts $\Delta_{\mathrm{FH}}$ at $X_{0}$ is calculated as

$$
\begin{aligned}
\Delta_{\mathrm{FH}} & \triangleq \mathbb{E}^{! o}\left[\sum_{i} a_{0} a_{i} \kappa_{0 i}\right] \\
& =\mathbb{E}\left[\sum_{i \neq 0} a_{0} a_{i} \kappa_{0 i}\right],
\end{aligned}
$$

where the expectation is over all uncertainties $a_{0}, \ldots, a_{N-1}$ and $\kappa_{0,1}, \ldots, \kappa_{0, N-1}$. The second equation follows by application of Slivnyak's theorem. Taking the expectation yields

$$
\begin{aligned}
\Delta_{\mathrm{FH}} & =\mathbb{E}\left[a_{0}\right] \sum_{i=1}^{N-1} \mathbb{E}\left[a_{i}\right] \mathbb{E}\left[\kappa_{0 i}\right] \\
& =\frac{\bar{a}^{2}}{M}(N-1),
\end{aligned}
$$

since $\mathbb{E}\left[\kappa_{0 i}\right]=1 \times \mathbb{P}\left\{X_{i}\right.$ chooses the same channel as $\left.X_{0}\right\}=$ $1 / M$ in the uncoordinated FH-CDMA case.

2) Case MLLO-FH-CDMA: Let again $\mathcal{G}_{k}$ denote the $k$ th layer in our simplified scenario, where $\mathcal{G}_{0}$ is the lowest layer. Since in every layer $\mathcal{G}_{k}$ the nodes have orthogonal channels, it is necessary to require that $\left|\mathcal{G}_{k}\right| \leq M$ for all $k$. Furthermore, the following assumptions concerning the scheduling algorithm are made:

\footnotetext{
${ }^{6}$ Due to lack of stationarity of the Binomial point process, the point $X_{0}$ is not typical in the sense that all other points have the same view of the process. However, since in our calculations distances will not be involved in any way, we are able to claim that the point $X_{0}$ will reflect the characteristics of all points of the process.

${ }^{7}$ This will not quantify the actual interference situation at the nodes but will allow a simple comparison of the two schemes. The analysis of the number of conflicts can be taken as a rough measure to quantify the improvement of MLLO hopping compared to uncoordinated FH-CDMA.
} 
- (i) At the time the algorithm has found a solution, the nodes from $\Pi$ are distributed to the layers $\mathcal{G}_{k}$ in a bottomup way, i.e., according to the sequential assignment rule $X_{i} \in \mathcal{G}_{k}$, if and only if $\left|\mathcal{G}_{0}\right|=\ldots=\left|\mathcal{G}_{k-1}\right|=M \wedge$ $\left|\mathcal{G}_{k}\right|<M$. We denote by $\mathcal{G}_{K}$ is the highest non-empty layer.

- (ii) The order of the assignments is assumed to be random. Hence, there is initially no incentive for a certain node $X_{i}$ to choose a certain layer $\mathcal{G}_{k}$ and the probability of the event $X_{i} \rightarrow \mathcal{G}_{k}$ is equal for all nodes $X_{i}$.

We consider again the average number of conflicts at the node $X_{0}$. After the scheduling process, two cases concerning the assignment of node $X_{0}$ to a layer $\mathcal{G}_{k_{0}}$ emerge, depending on whether the layer $\left|\mathcal{G}_{k_{0}}\right|<M$ or $\left|\mathcal{G}_{k_{0}}\right|=M$.

Case $\left|\mathcal{G}_{k_{0}}\right|=M$ : Here, we require that $N>=M$. Without loss of generality we label the remaining nodes within the same layer $\mathcal{G}_{k_{0}}$ by $X_{2}, \ldots, X_{M-1}$. These nodes do not create conflict to node $X_{0}$ and we write $\kappa_{0 i}=0$ for $i=1, \ldots M-$ 1. We now consider the other layers $\mathcal{G}_{k}$ for which $\left|\mathcal{G}_{k}\right|=$ $M$, according to (i). Here, in each of these layers there will always be a node creating a conflict to $X_{0}$, since in each of these layers, $M$ nodes are orthogonal on $M$ channels. If the highest layer $\mathcal{G}_{K}$ has cardinality smaller than $M$, there is no deterministic collision with node $X_{0}$. In fact, the probability of a collision depends on the number of members within this layer, according to

$$
\mathbb{P}\left\{\bigvee_{i: X_{i} \in \mathcal{G}_{K}}\left(\kappa_{0 i}=1\right)\right\}=\frac{\left|\mathcal{G}_{K}\right|}{M}
$$

Hence, we can write

$$
\begin{aligned}
\mathbb{E}^{! o}\left[\sum_{i} a_{0} a_{i} \kappa_{0 i}|| \mathcal{G}_{k_{0}} \mid=M\right] & \\
= & \sum_{\substack{k: \mathcal{G}_{k} \mid=M \\
k \neq k_{0}, K}} \mathbb{E}\left[a_{0}\right] \mathbb{E}\left[\bigvee_{i: X_{i} \in \mathcal{G}_{K}}\left(a_{i} \kappa_{0 i}=1\right)\right] \\
& +\mathbb{E}\left[a_{0}\right] \mathbb{E}\left[\bigvee_{i: X_{i} \in \mathcal{G}_{K}}\left(a_{i} \kappa_{0 i}=1\right)\right]
\end{aligned}
$$

With the above observations, we conclude that $\mathbb{E}\left[\vee_{i: X_{i} \in \mathcal{G}_{k}}\left(a_{i} \kappa_{0 i}=1\right)\right]=\mathbb{E}\left[a_{i}\right] \times 1$ and $\mathbb{E}\left[\vee_{i: X_{i} \in \mathcal{G}_{K}}\left(a_{i} \kappa_{0 i}=1\right)\right]=\mathbb{E}\left[a_{i}\right] \times\left|\mathcal{G}_{K}\right| / M$, so we can rewrite (5) as

$$
\mathbb{E}^{! o}\left[\sum_{i} a_{0} a_{i} \kappa_{0 i}|| \mathcal{G}_{k_{0}} \mid=M\right]=\bar{a}^{2}\left(\frac{N}{M}-1\right) .
$$

Case $\left|\mathcal{G}_{k_{0}}\right|<M$ : Here, conflicts deterministically emerge from the lower layers $\mathcal{G}_{k}$, since $\left|\mathcal{G}_{k}\right|=M$ for all $k<k_{0}$. We thus can write

$$
\mathbb{E}^{! o}\left[\sum_{i} a_{0} a_{i} \kappa_{0 i}|| \mathcal{G}_{k_{0}} \mid<M\right]=\bar{a}^{2}\left\lfloor\frac{N}{M}\right\rfloor .
$$

Using the assumption (ii), we can determine the probabilities of the two cases as follows: Given the final distribution of the nodes $X_{i}$ to the layers $\mathcal{G}_{k}$, the probability of node $X_{0}$ being in a layer $\mathcal{G}_{k}$ with $\left|\mathcal{G}_{k}\right|=M$ is equivalent to the probability of the event $\left|\mathcal{G}_{k_{0}}\right|=M$. Hence, we write

$$
\mathbb{P}\left\{\left|\mathcal{G}_{k_{0}}\right|=M\right\}=\frac{\text { Number of Possibilities }}{\text { Number of Nodes }}=\frac{M\left\lfloor\frac{N}{M}\right\rfloor}{N} .
$$

Accordingly, the complementary event has probability

$$
\mathbb{P}\left\{\left|\mathcal{G}_{k_{0}}\right|<M\right\}=1-\frac{M\left\lfloor\frac{N}{M}\right\rfloor}{N} .
$$

Combining (6)-(9), we conclude that

$$
\begin{aligned}
\Delta_{\mathrm{MLLO}} & \triangleq \mathbb{E}^{! o}\left[\sum_{i} a_{0} a_{i} \kappa_{0 i}\right] \\
& =\bar{a}^{2}\left(\left(\frac{N}{M}-1\right) \frac{M\left\lfloor\frac{N}{M}\right\rfloor}{N}\left\lfloor\frac{N}{M}\right\rfloor\left(1-\frac{M\left\lfloor\frac{N}{M}\right\rfloor}{N}\right)\right) \\
& =\bar{a}^{2}\left\lfloor\frac{N}{M}\right\rfloor\left(2-\frac{M}{N}\left(1+\left\lfloor\frac{N}{M}\right\rfloor\right)\right) .
\end{aligned}
$$

To analyze the performance of MLLO-FH-CDMA compared to uncoordinated FH-CDMA, the relative improvement in terms of reduction of average conflicts, i.e., $\eta=1-\frac{\Delta_{\mathrm{MLLO}}}{\Delta_{\mathrm{FH}}}$, is shown in Fig. 2. Note that the $\eta$ is independent of $\bar{a}$. For $N / M \leq 1$, the relative improvement is maximal, since all nodes are situated in one layer only and thus, are orthogonalized. As the ratio $N / M$ grows, the performance of MLLO becomes similar to that of uncoordinated FH-CDMA.

\section{Simulations}

To evaluate outage probabilities of MLLO-FH-CDMA in a large network, we need to make assumptions on the traffic model and geometry of the network.

We assume that communication partners are chosen uniformly within the neighborhood and that each node transmits with probability $\bar{a}$, reflecting the overall network activity. A packet is deemed decodable, if the SINR threshold $\beta>1$. The path loss exponent is assumed to be $\alpha=4$, fading is not considered $^{8}$.

Fig. 3 shows the resulting packet error rates, spatially interpolated and averaged over 10000 possible network states, of FH-CDMA and MLLO-FH-CDMA for realization of a Bernoulli point process with 150 nodes using $M=50$ channels on a disc with $R_{\mathrm{sim}}=100$. All nodes within $r=25$ units of each other are assumed to be in a neighborhood. Fig. 4 shows the corresponding sorted outage probabilities for the same spatial configuration and various activity levels. The threshold values $\tau$ were chosen as $0.05,0.03$, and 0.01 , respectively. As can be seen, the absolute gain of MLLO-FHCDMA increases with network activity, while the relative gain is approximately constant. The outage probability is especially reduced in the center of the network, where the overall interference temperature is high in case of uncoordinated FHCDMA.

\footnotetext{
${ }^{8}$ Including fading effects in the simulation does not change the behavior,
} MAI is reduced on average. 


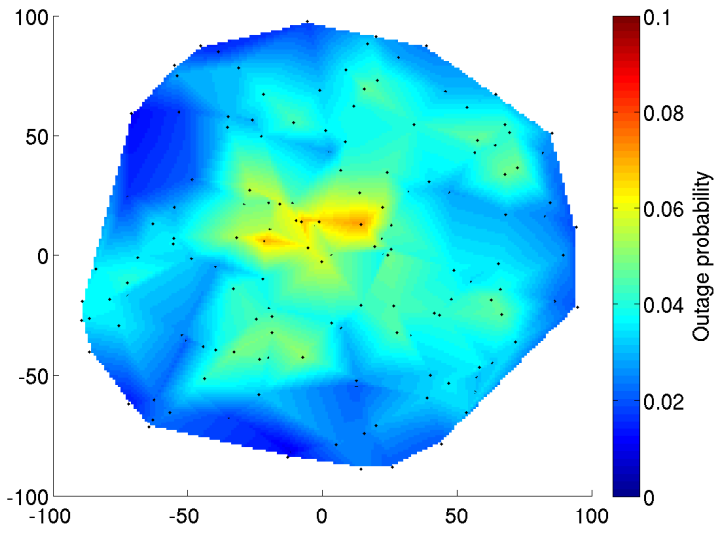

(a) Standard FH-CDMA

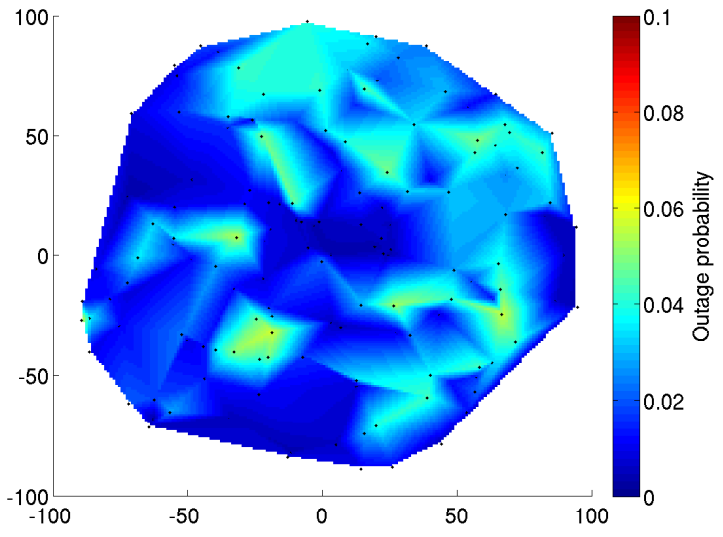

(b) MLLO-FH-CDMA

Fig. 3. Resulting outage probabilities for a scenario of 150 nodes.

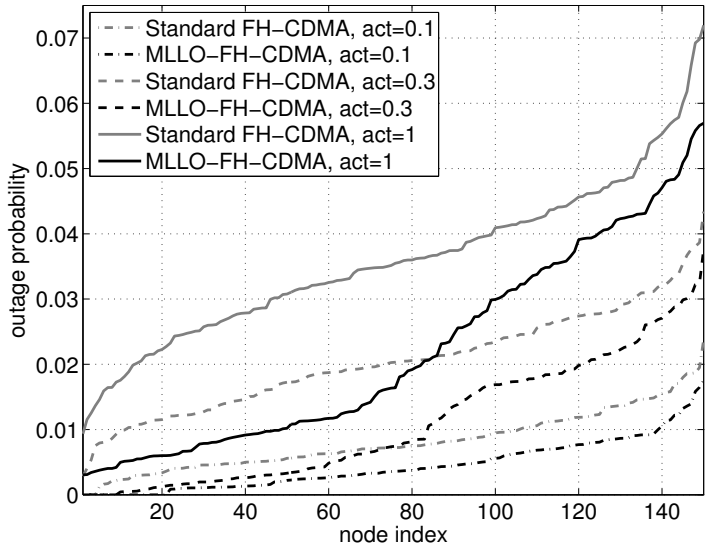

Fig. 4. Ordered outage probabilities for the spatial configuration shown in Fig. 3.

\section{CONCLUSION AND FUTURE WORK}

We introduced multi-level locally orthogonal FH-CDMA as a method to reduce MAI in large scale ad hoc networks. A possible algorithm to implement MLLO hopping has been given and verified in with simulations.

We assume that the gains largely depend on the traffic patterns of the nodes and the geometry. Geometrically clustered and very active interference limited networks will likely benefit most by introducing the geometrical ordering through hopping layers. Hence, we will focus in future work on the analysis of MLLO-FH-CDMA and comparison with different orthogonal hopping schemes both on the physical layer and on the MAC layer. For the analysis of the interference field, we are working on extending prior work [11], [12], [13], [4], focused on modeling FDMA in a Poisson point process geometry model. For evaluation of the effects on the MAC layer, we are aiming to apply the comparison framework of Mo et al. [5].

We believe that especially in the protocol design of large frequency hopping multi-channel ad hoc networks, where self- interference is a limiting factor to performance, the application of MLLO hopping should be considered.

\section{REFERENCES}

[1] J. Zander and G. Malmgren, "Adaptive frequency hopping in HF communications," Communications, IEE Proceedings-, vol. 142, no. 2 , pp. 99-105, Apr 1995.

[2] P. Poposki, H. Yomo, and R. Prasad, "Strategies for adaptive frequency hopping in the unlicensed bands," IEEE Wireless Communications, Dec 2006.

[3] P. Popovski, H. Yomo, and R. Prasad, "Dynamic adaptive frequency hopping for mutually interfering wireless personal area networks," IEEE Transactions on Mobile Computing, vol. 5, pp. 991-1003, 2006.

[4] R. Tanbourgi, J. P. Elsner, H. Jaekel, and F. K. Jondral, "Bounds on the second reduced moment measure for the analysis of FDMA scheduled ad hoc networks," in Spatial Stochastic Models for Wireless Networks (SpaSWiN'11).

[5] J. Mo, H.-S. W. So, and J. Walrand, "Comparison of multichannel mac protocols," IEEE Transactions on Mobile Computing, Jan 2008.

[6] J. Lee, J. Mo, M. T. Trung, J. Walrand, and W. H.-S. So, "Design and analysis of a cooperative multichannel mac protocol for heterogeneous networks," IEEE Transactions on Vehicular Technology, Sep 2010.

[7] R. Brooks, "On colouring the nodes of a network," Mathematical Proceedings of the Cambridge Philosophical Society, Apr 1941, doi:10.1017/S030500410002168X.

[8] D. J. Leith and P. Clifford, "Convergence of distributed learning algorithms for optimal wireless channel allocation," in in Proceedings of IEEE Conference on Decision and Control, 2006, pp. 2980-2985.

[9] K. R. Duffy, N. O’Connell, and A. Sapozhnikov, "Complexity analysis of a decentralised graph colouring algorithm," Inf. Process. Lett., vol. 107, no. 2, pp. 60-63, 2008.

[10] A. Baddeley, "Spatial point processes and their applications," in Stochastic Geometry: Lectures given at the C.I.M.E. Summer School held in Martina Franca, Italy, September 13-18, 2004, ser. Lecture Notes in Mathematics 1892 (subseries: Fondazione C.I.M.E., Firenze), A. Baddeley, I. Bárány, R. Schneider, and W. Weil, Eds. Springer Verlag, 2006, pp. 1-75, ISBN 3-540-38174-0.

[11] J. Elsner, R. Tanbourgi, and F. Jondral, "On the Transmission Capacity of Wireless Multi-Channel Ad Hoc Networks with local FDMA scheduling," in International Congress on Ultra Modern Telecommunications and Control Systems, Oct 2010.

[12] _ "A Note on Local Receive Channel Scheduling versus Transmit Channel Scheduling in Wireless Multi-Channel Ad Hoc Networks," in International Conference on Computer and Information Application, Dec 2010.

[13] R. Tanbourgi, J. Elsner, and F. Jondral, "Lowering Outage Probability in Ad Hoc Networks by Nearest Neighbor FDMA Scheduling," in IEEE WCNC 2011, Cancun, Mexico, Mar 2011. 